\title{
Enhancing QoS in Mobile Learning Multimedia in Resource Constrained Environments
}

\author{
Nazir Ahmad Suhail \\ East African University \\ RwandaNyagatare, Rwanda
}

\begin{abstract}
The popularity of mobile learning multimedia applications is increasing day by day. However, the provision of guaranteed Quality of Service (QoS) in mobile learning multimedia in developing countries is still challenging due to scarcity of link bandwidth. This paper presents a model that enhances QoS in mobile learning experience enriched with multimedia, aimed to increase the level of user satisfaction with a focus on resource constrained environments. The paper achieved the goal by using optimization technique on both communication network and multimedia contents to reduce information loss, latency, and jitter with improved audiovisual quality among others.
\end{abstract}

\section{General Terms}

Network Optimization, Multimedia Optimization.

\section{Keywords}

Mobile Learning Multimedia, Quality of Service (QoS), QoS Framework, Resource Constrained Environments.

\section{INTRODUCTION}

The turn of the new century has witnessed a clear shift from fixed telephony to cellular phones. Wireless communication is changing paradigm from voice-alone services to the new world of real-time multimedia applications [1]. Advances in wireless communication technologies and mobile networks that use a range of multimedia applications have brought about remarkable changes in the people's daily lives around the world. Mobile devices are seen as the fastest spreading technology in human history. These devices changed the way we communicate and function in society. Such technological advancements have created new educational models such as mobile learning that are embedded into mobile life in formal and in informal educational settings. Mobile learning has some unique characteristics that include: flexibility of location and learning time, interactive knowledge acquisition, adaptation to learner's needs, ubiquitous learning environment, and integrated instructional context efficiency for both re-use and feedback [2].

Mobile learning can be used effectively in distance learning environment, especially in the universities in developing countries to meet the growing demand for higher education. Using multimedia technologies to prepare the learning content, instructional designers can enrich the learning content with more than one media such as video, audio, graphics and text to provide an efficient and pleasant distance learning experience to users through mobile technologies. However, the mobile users may face challenges of congestion that causes delay [3] due to limitation of available wireless bandwidth which is always scarce in developing countries. At the same time, multimedia QoS will also be reduced significantly due to the constraints in network resources. The provision of QoS for mobile learning multimedia applications imposes a major challenge that requires investigation because of low bandwidth environment and mobility of portable devices in developing countries.

Therefore, mobile communication network that support multimedia entails substantial efforts for provision of guaranteed Quality of Service (QoS) to users through efficient and effective utilization of network resources that require new resource management techniques [4].

The scarcity of link bandwidth in wireless networks and provision of guaranteed QoS in multimedia communication, demands to develop a robust mechanism for improving QoS in multimedia applications for mobile learning [5]. The new generation of mobile communication networks are expected to provide different levels of guaranteed QoS for a wider spectrum of wireless applications and users with extremely varied requirements [6] that requires to bring network optimization techniques into sharp focus [7] aimed to enhance application performance. On the other hand, this research argues that to achieve the goal, instead of optimizing only communication network, multimedia application should also be optimized alongside, due to the fact that they are symbiotic [8].

Motivated by the facts presented above and to solve the problem effectively, this paper formulates a novel QoS provisioning framework that optimizes network resources and multimedia application in an effort to enhance the efficiency of multimedia content for mobile learning that can increase the level of user satisfaction with minimal available network resource amidst the stringent bandwidth requirements in the context of resource constraint environments.

This paper is organized as follows: Section 3 presents the Mobile Learning Multimedia QoS Framework for Resource Constrained Environments discussed under the themes Network Optimization and Multimedia Optimization in detail. Finally, after short discussion, a conclusion is drawn and future work is also highlighted in section 4 .

\section{TERM DEFINITIONS}

\subsection{Mobile learning}

Mobile learning is a new learning environment which is still at infancy stage and evolving. Therefore, there is no generally agreed upon definition of the term, different authors define it differently in many different ways. However, one common concept in all the definitions is that mobile learning is learning experience supported by hand held devices such as personal digital assistants (PDAs), mobile phones or wireless laptop PCs. 


\subsection{Quality of Service (QoS)}

QoS as defined by [9] is the perceived level of user satisfaction resulted by "a collective effect of service performance" by multimedia application.

\section{MOBILE LEARNING MULTIMEDIA QOS FRAMEWORK for RESOURCE CONSTRAINED ENVIRONMENTS}

It is a common and fair assumption from many first world researchers that mLearning is not really possible in rural settings, where low bandwidth restricts the use of bandwidth intensive resources such as video-streaming, multimedia and operating rich applications [10]. But there have been many successful mLearning projects in deep rural settings on some of the most basic mobile phones and other mobile devices.

It is a well known fact that capabilities of multimedia applications in mobile devices have evolved significantly in the recent years and they will continue to evolve further in the future times to come. However, enabling multimedia applications over Internet connections in developing countries suffers significantly due to low Internet bandwidth. To address the issue this paper proposes a framework to enhance multimedia QoS for mobile devices for users in resource constrained environments. Fig 1 presents the Model that has two main components discussed under the titles Network Optimization and Multimedia Optimization in the following sections. Network and Multimedia optimization processes are governed by Quality of Service (QoS) Rules of both components. The output of network optimization results into high throughput, reduced information loss, reduced latency, and low jitter. Whereas, the output of multimedia optimization produces high quality data stream with attributes such as small size multimedia file, low error rate, improved audio visual quality, data with no redundancy, and perceived end user quality. The interaction between above works as input to produce enhanced QoS mobile learning multimedia for resource constrained environments to facilitate mobile learning as shown in the figure.

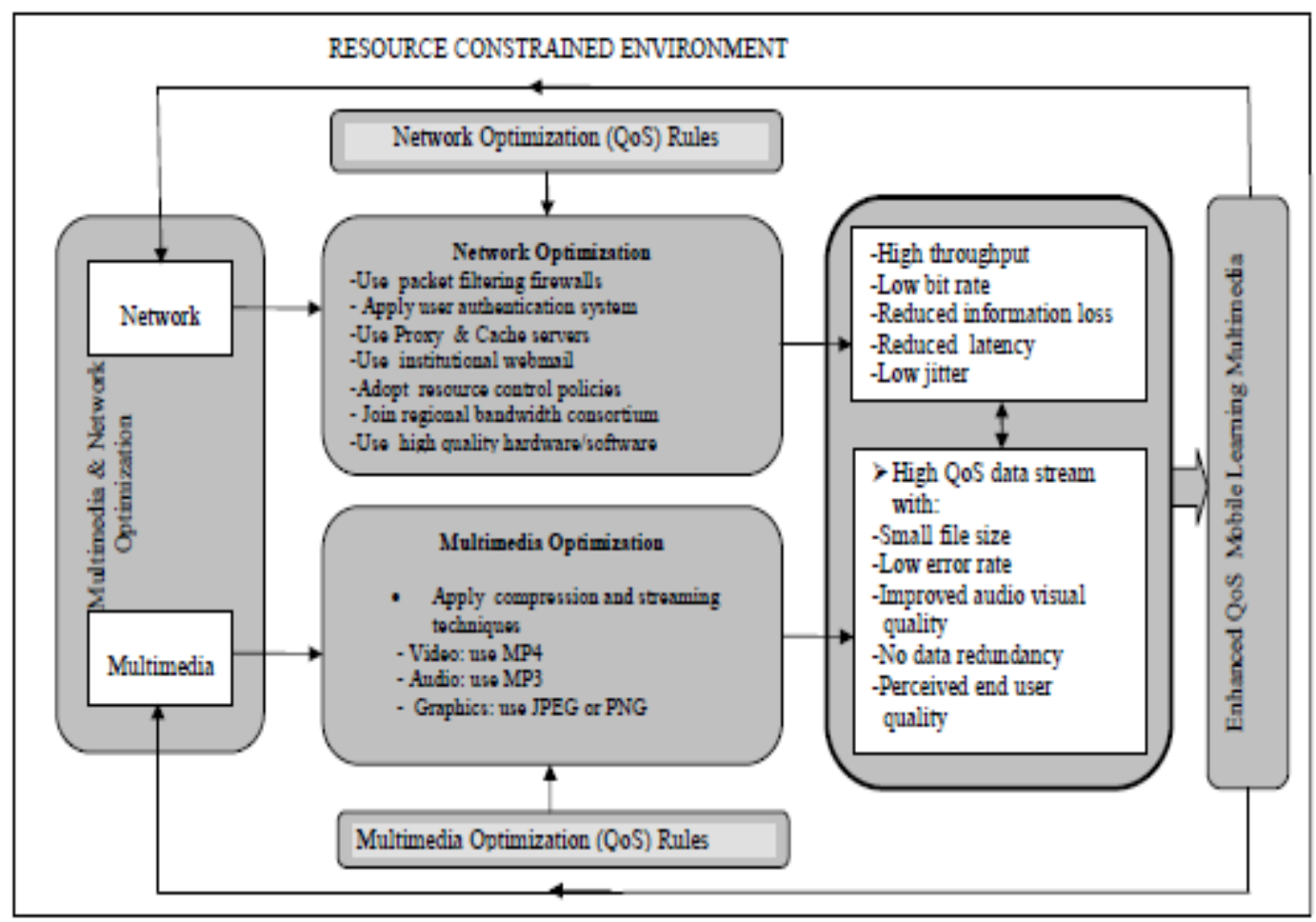

Fig 1: Mobile Learning Multimedia QoS Framework for Resource Constrained Environments

\subsection{Network Optimization}

The rapid evolution in the mobile network subscribers requires more efficient network technologies that can provide faster data communication services to the users. Multimedia transmission mobile networks need to meet the requirements such as high bandwidth, low error rate, low delay, and low jitter [5]. Various techniques can be used to increase the efficiency of mobile networks.

\subsubsection{Filtering the Network Traffic:}

Network filtering can be applied in mobile communication networks effectively by using firewalls and Intrusion Prevention Devices (IPS) on the border of the Internet [11]. A firewall is a device comprised of hardware and software used to enhance security in a network system by applying a security policy governing the flow of traffic between two or more networks [12]. Information on Internet travels in form of discrete packets between networks. The basic functionality of all firewall devices is filtering incoming and outgoing packets. Packet filtering process is done by configuring filtering rules, namely, Access Control Lists (ACLs) [13] on routers and/or firewall devices.

\subsubsection{Access Control lists (ACLs):}

$A$ network access control list (ACL) is an optional security layer that behaves as a firewall used for controlling in and out traffic of a subnet. ACLs technology plays an important role to optimize network performance and secure the network against threats with minimal costs. An ACL is a set of rules applied in a sequential manner intended to implement security objectives that determines the access rights for every packet based on information reflected at the header field of arriving 
at the firewall, originating from different users and network resources. The ACL filters the traffic and determines whether to forward or discard a packet. The information classifying packets include; protocol field, source IP address, destination IP address, source port, destination port, TCP flags, Internet Control Message Protocol (ICMP) type and ICMP code [14].

\subsubsection{Time Based ACLs}

Time based ACLs are just like normal ACLs used to restrict the access of network resources for some specific time range when they become active, mostly during peak working hours. To minimizes the bandwidth usage that be can be used for academic purposes. The end time of such ACLs remains valid until the beginning of next minute, e.g., to make ACL to be active from 11:00 pm to 2:00 am we need to define the time as follows $>21: 00$ to 01:59

- Time-based ACLs are nested within Named or Numbered ACLs. It is possible have multiple time-based Access Control Entries (ACE) in an ACL.

Time-Based ACLs are configured on router interfaces in the network to restrict the heavy file download timings to save the bandwidth.

\subsubsection{Traffic Shaping and Prioritization}

Prioritization technique is used to enhance the Quality of Service $(\mathrm{QoS})$ for real time applications such as voice, video or other multimedia traffic, are processed ahead of other types of traffic. In a similar manner ACLs are used to prioritize the request made by various categories of users based upon their associated privileges ([14], [15]).

\subsubsection{Authentication}

With tremendous increase in computing resources, usage of authentication systems has become vital, whereby password based authentication mechanism is one of the simplest and the most convenient networks authentication technique over insecure networks. Authentication system provides access to only authorized users to use the network resources. Password type authentication is more frequently required in areas such as wireless networks and computer networks. Research shows that authentication schemes that use smart cards has also attracted the attention of many scholars [16].

\subsection{Multimedia Optimization}

Ubiquitous provision of wireless services that are rich in multimedia, in cellular networks call for larger amounts of bandwidth which is insufficient in resource constrained environments. The fundamental characteristics of multimedia applications are that they are bandwidth hungry and generally consume megabits per second instead of kilobits per second. However, the QoS in mobile applications can be enhanced significantly by using multimedia streaming and compression techniques to address the issue [15].

\subsubsection{Multimedia Streaming}

Streaming Media that refers transmitting real time video and audio files to the receiver in form of a continuous stream once the sender starts transmitting contents, can provide QoS for live and on-demand to the iPhone, iPad, iPod Touch, Android and other mobile devices. However, the streaming media must be compressed (coded) before process of streaming begins as such files are heavy. At the same time it requires that the coded files are decoded (decompressed) before they a are viewed by the receiver.

\subsubsection{Multimedia Compression}

Quality of Service (QoS) is a key factor in distributed multimedia systems. Transmitting multimedia data, visual media (video, audio and graphics) in particular to users over mobile networks demands for larger amounts of bandwidth which is limited in low bandwidth environment. Data compression technique significantly reduces the file size. The compression is of two types, namely lossy and lossless. In lossless compression, the integrity of original data is preserved and full data is recovered after decoding (decompression) while in lossy compression (mostly used for video and audio files) it is not true. The focus of this research is on video, audio, and graphics although text is also part of multimedia.

\section{Video}

Video is a time sensitive multimedia format that demands for high bandwidth, the large size storage space as compared to other multimedia forms such as audio, graphics and text.

Several studies (e.g., [17], [19]) conclude that MPEG-4 algorithm provides efficient compression as compared with other video formats. MPEG-4 has the potential to improve perceived video quality, especially video telephony and streaming video at bit rates as low as $64 \mathrm{kbps}$.

\section{Audio}

The recent study [18] results identified Moving Pictures Layer (MP3) as most appropriate multimedia audio format for mobile learning in resource constraint environments. MP3 can support academic processes efficiently and effectively. MP3 is non proprietary, can be used across multiple platforms, and is approved by International Standards Organization (ISO).

\section{Graphics}

The most commonly used graphic formats include: Bitmap Image File, Graphic Interleave File, Tagged Image file, Portable Network Graphic, and Joint Pictures Expert Group. The research ([17],[18]) shows that Portable Network Graphics (PNG) and Joint Pictures Expert Group (JPEG) are ISO approved, open source products that can be used across multiple platforms. JPEG is created by Joint Photographic Experts Group- an ISO group of experts that develops and maintains standards for compression algorithms of image files.

\section{CONCLUSION}

This paper proposed the Mobile Learning QoS Framework that potentially reduces network resource consumption, alleviates network congestion within the bandwidth constrained environment. According to the framework, low bandwidth cannot restrict the use of bandwidth intensive resources such as video streaming and other rich applications in low bandwidth environment. The proposed solution is simple yet effective, and can be implemented on mobile platforms. The framework used optimization technique on multimedia and network to enhance QoS in mobile learning multimedia in the context of emerging economies where link bandwidth is insufficient as compared to the needs. Future work may include the validation of the model through experimental scientific research process. 


\section{REFERENCES}

[1] Ajina, A, Nair, M. K. 2015. A study of QoS Algorithm forNetworkingandMobileComputing,InternationalJourna lofCoputer Science Engineering,vol.4, no.4 2015, pp. 203-207.

[2] Wu, Tin-Yu; Chao, Han-Chieh, 2008. Mobile eLearningfor Next Generation Communication Environment,International Journal of Distance Education Technologies, 6(4), Oct-Dec 2008, pp.1-13.

[3] Ravindranath, K., Rao, K.R.S. 2013. A Survey on Energy aware offloading Techniques for Mobile CloudComputing, International Journal of Computer Trendsand Technology (IJCTT), vol.4, Issue 7-July, 2013, pp. 2081-2086.

[4] Ayyappan, K., Kumar, R. 2010. QoS CapacityEnhancement for WCDMA Networks with CodingScheme, International Journal of VLSI Design andCommunication System, vol.1, no.1, 2010.

[5] Luo, H., and Shyu, M-L. 2011. Quality of serviceprovision in mobile multimedia - a survey, Human-centric Computing and Information Sciences, 2011, vol.1 no.5doi:10.1186/2192-1962-1:5.

[6] Zhang] Xi , Cheng, W., Zhang, H. 2014. Heterogeneousstatistical QoS provisioning over 5G mobile wirelessnetworks, Network, IEEE, 2014,vol.28, issue: 6 , pp.46-53.

[7] ITU 2010. "Measuring the Information Society, 2010". [Online] Available: http://www.itu.int/ITUD/ict/statistics/material/graphs/2010/Global_ICT_Dev_0 0-10.jpg

[8] Understanding Network-Transparent Application Acceleration and WAN Optimization. Cisco whitepaper, 2007. Cisco systems, Inc.

[9] ITU-TS. 1988, "Terms and Definitions Related to Qualityof Telecommunication Services," Recommendation.

[10] Elias, T. 2011. Universal Instructional DesignPrinciples for Mlearning, The International Review ofResearch in Open and Distance Learning 2011,vol.12,no.2.http://www.irrodl.org/index.php/irrodl/ article /view/965/179

[11] Oğul,M and Baktır,S. 2013. Practical Attacks on MobileCellular Networks and Possible Counter measures, Future Internet 2013, 5, 474-489. doi:10.3390/fi5040474.

[12] Acharya, S., Wang, J.,and Ge, Z. 2006 "Simulationstudy of firewalls to aid improved performance," A Greenberg Proceedings of 39th Annual Symposium onSimulation,April 2-6, 2006.

[13] Bensefia , H., and Ghoualmi N. 2011. An intelligent system for decision making in firewallforensics, International Journal of Digital Informationand Communication Technology and Its Applications, 2011 vol. 166, pp. 470-484.

[14] Kaminsky, M. (2004).User Authentication and Remote Execution Across Administrative Domains. Doctor ofPhilosophy Thesis, Massachusetts Institute.

[15] Suhail, N.A., Lubega, J. 2011. Optimization Technique for Implementation of Blended Learning in ConstrainedLow Bandwidth Environment Lecture Notes inComputer Science, Springer, Heidelberg, 2011, vol. 348, pp. 166-173.

[16] H.-Y. Chien, J.-K. Jan, Y.-M. Tseng, An efficient and practical solution to remote authentication: Smart card,Computers \& Security 21, 2002, pp. 372-375.

[17] Suhail,N.A , Lubega, J, and Maiga, G. 2012. Multimedia to Enhance Blended Learning Experiencein Constrained Low Bandwidth Environment,LectureNotes in Computer Science, Springer,Heidelberg 2012, vol. 7411 of the series pp 362-372.

[18] Suhail, N., A.2015. A Multilevel Systematic Approach for Identification of Mobile Learning Multimedia in LowBandwidth Environment. International Journal ofComputer Applications (0975 - 8887) vol.119, no.17, June 2015.

[19] Liang, J. 1999. New Trends in Multimedia Standards: MPEG4 and JPEG. Info. Sc. Special issue on multimedia informing technologies-part 1, vol. 2 (4), 1999. 